\title{
Frail by different measures: a comparison of 8-year mortality in The Irish Longitudinal Study on Ageing (TILDA)
}

\author{
Roman Romero-Ortuno ${ }^{1,2,3}\left[\right.$ D Peter Hartley ${ }^{1,2,4} \cdot$ Rose Anne Kenny ${ }^{1,2,3} \cdot$ Aisling M. O'Halloran ${ }^{1,2}$
}

Received: 16 August 2021 / Accepted: 23 September 2021 / Published online: 1 November 2021

(c) The Author(s) 2021

\section{Key summary points}

Aim To compare how four different frailty classifications predicted 8-year mortality in TILDA.

Findings In those aged 65 or more years, frailty prevalences were $3.7 \%$ by FRAIL, $6.7 \%$ by FP, $16.6 \%$ by CFS, and $22.0 \%$ by FI. Mortality proportions were $57.1 \%, 57.8 \%, 36.8 \%$ and $35.6 \%$, respectively.

Message All tools significantly predicted mortality, but FRAIL and FP seemed more specific.

\begin{abstract}
Purpose We compared the ability of four frailty identification tools (frailty phenotype: FP; FRAIL scale; 32-item Frailty Index: FI; and Clinical Frailty Scale: CFS) to predict 8-year mortality in TILDA.

Methods We included wave 1 (2010) participants with data for all four tools. Mortality was ascertained at wave 5 (2018). Age, sex and education-adjusted binary logistic regression models were computed.

Results At baseline, there were 5700 participants (mean age 63, range 50-98, 54\% women). Frailty prevalences were 2.3\% by FRAIL, $3.8 \%$ by FP, $10.9 \%$ by CFS, and $12.8 \%$ by FI. Mortality was $41.2 \%, 44.9 \%, 25.3 \%$ and $27.0 \%$, respectively. The highest adjusted OR for mortality was for FRAIL (OR 4.48, 95\% CI 2.93-6.85, $P<0.001$ ), followed by FP (OR 3.55, 95\% CI 2.52-5.00, $P<0.001$ ), FI (OR 2.10, 95\% CI 1.68-2.62, $P<0.001$ ), and CFS (OR 1.88, 95\% CI 1.48-2.38, $P<0.001$ ).

Conclusions All tools significantly predicted mortality, but FRAIL and FP seemed more specific.
\end{abstract}

Keywords Frailty $\cdot$ Mortality $\cdot$ Comparative study $\cdot$ Longitudinal study $\cdot$ Older people

\section{Introduction}

Frailty is a state of dysregulation in multiple physiological systems and vulnerability to stressors [1]. Many frailty identification tools exist that differ in their conceptualisation vis-à-vis morbidity, disability, cognition, and other geriatric assessment dimensions [2].

Roman Romero-Ortuno

romeroor@tcd.ie

1 The Irish Longitudinal Study on Ageing (TILDA), Trinity College, Dublin, Ireland

2 Discipline of Medical Gerontology, School of Medicine, Trinity College, Dublin, Ireland

3 Mercer's Institute for Successful Ageing, St James's Hospital, Dublin, Ireland

4 Department of Public Health and Primary Care, University of Cambridge, Cambridge, UK
Several population-based studies have compared frailty identification tools for the prediction of mortality, including the Health and Retirement Study (HRS) [3] and the National Health and Nutrition Examination Survey (NHANES) [4] in the USA; and, in Europe, the Survey of Health, Ageing and Retirement in Europe (SHARE) [5], the Tree-City study [6], and the English Longitudinal Study of Ageing (ELSA) [7]. These are among the many studies that have compared the performance of different frailty tools in their prediction of adverse health-related outcomes in different populations.

The present study added value by comparing the ability of four different frailty identification tools to predict 8-year mortality in The Irish Longitudinal Study on Ageing (TILDA). 


\section{Methods}

\section{Design and setting}

We analysed data from TILDA, a population-based longitudinal study that collects information on the health, economic and social circumstances from people aged 50 and over in Ireland. Wave 1 of the study (baseline) took place between October 2009 and February 2011, and subsequent data were collected approximately 2-yearly over four longitudinal waves (wave 2: February 2012 to March 2013; wave 3: March 2014 to October 2015; wave 4: January to December 2016; wave 5: January to December 2018). The full cohort profile has been described elsewhere [8]. All performance-based health measures in TILDA were collected by trained research nurses following standard operating procedures.

\section{Participants}

We included TILDA wave 1 participants aged 50 years or over who had complete data for frail state classification according to four frailty identification tools.

\section{Measures}

Frail state was defined as per the following four tools:

- Fried's physical frailty phenotype (FP): frail if $\geq 3$ features present. The operationalisation of the frailty phenotype in TILDA was the same as in the Cardiovascular Health Study [9], except for the physical activity criterion, for which we used the short form of the International Physical Activity Questionnaire (IPAQ) [10].

- Morley's FRAIL scale (frail if $\geq 3$ among fatigue, resistance, ambulation, illnesses, and loss of weight). This was operationalised in TILDA as previously described [11].

- A 32-item Frailty Index (FI $\geq 0.25$ ), the description of which has been detailed elsewhere [12],

- and the Clinical Frailty Scale classification tree (CFS $\geq 5$ ), as detailed elsewhere [13].

Age, sex and education were collected at baseline. The latter was defined as a three-level ordinal variable: up to primary (reference category), secondary and higher.

Mortality was ascertained for all study participants at each follow-up wave, following procedures described elsewhere [14].

\section{Statistical analyses}

All statistical analyses were carried out using IBM SPSS Statistics for Windows (Version 26.0. Armonk, NY: IBM Corp). Descriptives were given as mean with standard deviation (SD) and range, or count with percentage (\%). Overlap between the four frailty classifications was visualised by means of a Venn diagram created with the ggvenn package in $\mathrm{R}$ (version 0.1.9; https://CRAN.R-project.org/ package $=$ ggvenn). Sensitivity and specificity for 8 -year mortality were calculated for each frailty tool using MedCalc Software Ltd. Diagnostic test evaluation calculator, https://www.medcalc.org/calc/diagnostic_test.php (Version 20.009; accessed August 3, 2021). In addition, binary logistic regression models controlling for age, sex and education were computed for the extraction of odds ratios (OR) and $95 \%$ confidence intervals (CI). We did not control those models for multimorbidity or baseline physical activity because these were already included in some frailty definitions. The level of statistical significance was set at $p<0.05$. A sensitivity analysis was performed on the baseline sample aged 65 and over.

\section{Ethics}

Ethical approval for each wave was obtained from the Faculty of Health Sciences Research Ethics Committee at Trinity College Dublin, Ireland. All participants provided written informed consent.

\section{Results}

At wave 1, there were 5700 participants (mean age 63, SD 9.3, range 50-98 years, $54 \%$ women, $26 \%$ with primary education or less, and $33 \%$ with higher education) with data for frailty classification according to all four tools. The prevalences of frailty were $2.3 \%(N=131)$ by FRAIL, $3.8 \%(N=214)$ by FP, $10.9 \%(N=621)$ by CFS, and $12.8 \%$ $(N=729)$ by FI. Only 57 participants $(1 \%)$ were classified as frail by all tools. The vast majority of wave 1 participants (4634 or $81.3 \%$ ) were not classified as frail by any of the tools. Figure 1 shows the overlap between the four frailty classifications. Figure 2 shows the overlap in those aged 65 and over.

The 8-year mortality proportions (in those aged $\geq 50$ ) were $44.9 \%$ by FP, $41.2 \%$ by FRAIL, $27.0 \%$ by FI, and $25.3 \%$ by CFS. The $1 \%$ classified as frail by all tools had a mortality proportion of $43.9 \%$. The mortality for the $81.3 \%$ 

ing the overlap between the four frailty classifications in the first wave of TILDA (population aged 50 and over, $N=5700$ ). Number and percentages of TILDA wave 1 participants are shown. 4634 participants were not classified as frail by any scheme

Fig. 2 Venn diagram representing the overlap between the four frailty classifications in the first wave of TILDA (population aged 65 and over, $N=2289$ ). Number and percentages of TILDA wave 1 participants are shown. 1599 participants were not classified as frail by any scheme
Fig. 1 Venn diagram represent-
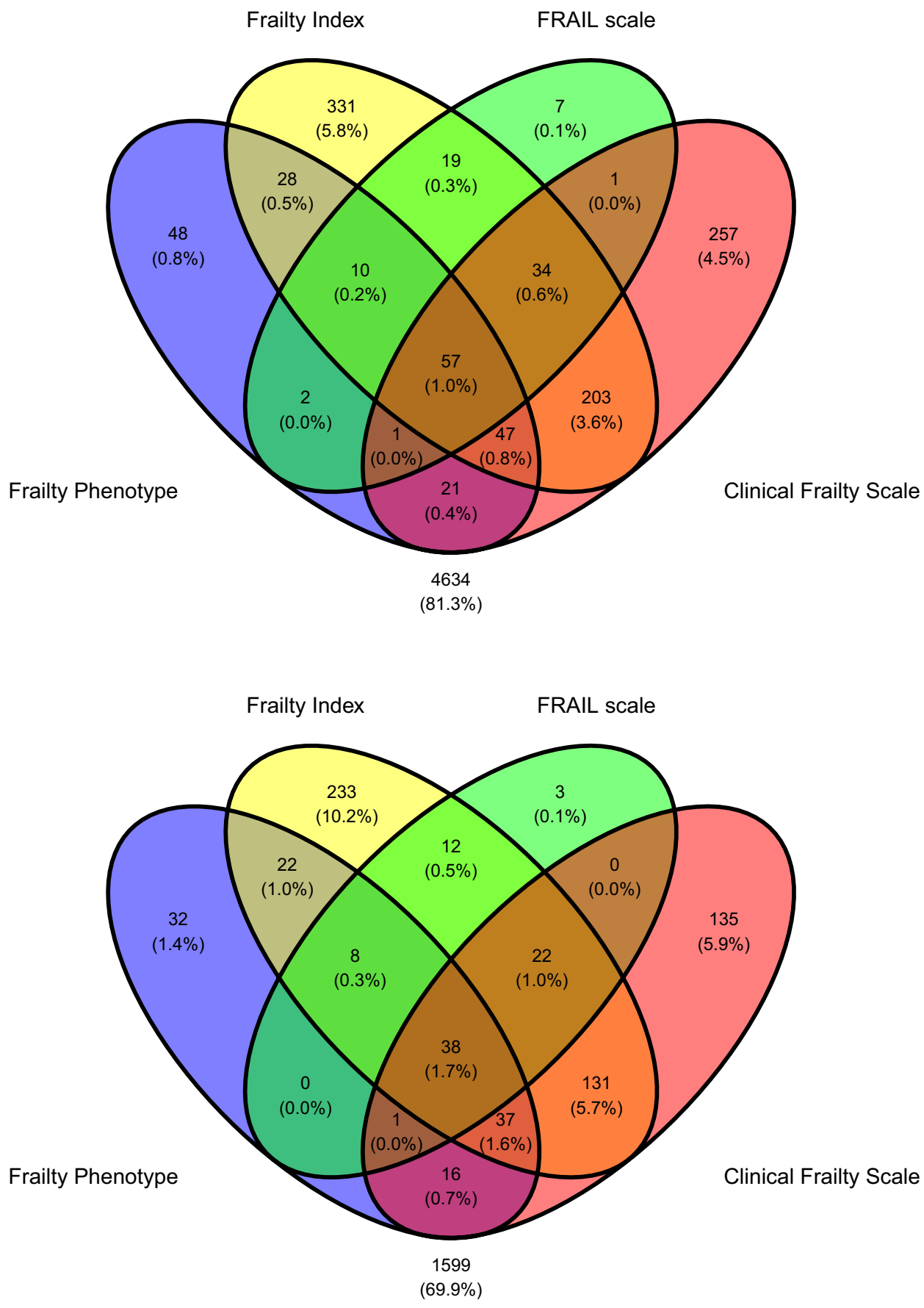

Table 1 Comparison of 8-year mortality proportions and binary logistic regression results (population aged 50 and over, $N=5700)$

\begin{tabular}{llllll}
\hline & $\begin{array}{l}\text { \% 8-year } \\
\text { mortality }\end{array}$ & $\begin{array}{l}\text { OR (age, sex, and } \\
\text { education-adjusted) }\end{array}$ & $\begin{array}{l}\text { Lower 95\% } \\
\text { CI for OR }\end{array}$ & $\begin{array}{l}\text { Upper 95\% } \\
\text { for OR }\end{array}$ & $P$ \\
\hline Frail by FRAIL $(N=131)$ & 41.2 & 4.48 & 2.93 & 6.85 & $<0.001$ \\
Frail by FP $(N=214)$ & 44.9 & 3.55 & 2.52 & 5.00 & $<0.001$ \\
Frail by FI $(N=729)$ & 27.0 & 2.10 & 1.68 & 2.62 & $<0.001$ \\
Frail by CFS $(N=621)$ & 25.3 & 1.88 & 1.48 & 2.38 & $<0.001$ \\
Frail by all $(N=57)$ & 43.9 & 3.92 & 2.05 & 7.50 & $<0.001$ \\
Frail by none $(N=4634)$ & 7.0 & 0.46 & 0.38 & 0.57 & $<0.001$ \\
\hline
\end{tabular}

$O R$ odds ratio, $C I$ confidence interval, $F P$ frailty phenotype, FI Frailty Index, CFS Clinical Frailty Scale 
Table 2 Comparison of 8-year mortality proportions and binary logistic regression results (population aged 65 and over, $N=2289$ )

\begin{tabular}{llllrl}
\hline & $\begin{array}{l}\text { \% 8-year } \\
\text { mortality }\end{array}$ & $\begin{array}{l}\text { OR (age, sex, and } \\
\text { education-adjusted) }\end{array}$ & $\begin{array}{l}\text { Lower 95\% } \\
\text { CI for OR }\end{array}$ & $\begin{array}{l}\text { Upper 95\% } \\
\text { for OR }\end{array}$ & $P$ \\
\hline Frail by FRAIL $(N=84)$ & 57.1 & 5.07 & 3.08 & 8.34 & $<0.001$ \\
Frail by FP $(N=154)$ & 57.8 & 3.47 & 2.38 & 5.06 & $<0.001$ \\
Frail by FI $(N=503)$ & 35.6 & 2.12 & 1.66 & 2.72 & $<0.001$ \\
Frail by CFS $(N=380)$ & 36.8 & 1.83 & 1.40 & 2.39 & $<0.001$ \\
Frail by all $(N=38)$ & 63.2 & 4.79 & 2.28 & 10.09 & $<0.001$ \\
Frail by none $(N=1599)$ & 14.9 & 0.47 & 0.37 & 0.60 & $<0.001$ \\
\hline
\end{tabular}

Table 3 Diagnostic evaluation of the four frail classifications for the prediction of 8-year mortality in TILDA (population aged 50 and over, $N=5700$ )

\begin{tabular}{|c|c|c|c|c|}
\hline & Value & $95 \% \mathrm{CI}$ & Value & $95 \% \mathrm{CI}$ \\
\hline & \multicolumn{2}{|l|}{ FRAIL } & \multicolumn{2}{|c|}{ Frailty phenotype } \\
\hline Sensitivity & $9.14 \%$ & $6.94-11.75 \%$ & $16.24 \%$ & $13.36-19.47 \%$ \\
\hline Specificity & $98.49 \%$ & $98.12-98.81 \%$ & $97.69 \%$ & $97.24-98.08 \%$ \\
\hline Positive likelihood ratio & 6.06 & $4.33-8.49$ & 7.03 & $5.45-9.08$ \\
\hline Negative likelihood ratio & 0.92 & $0.90-0.95$ & 0.86 & $0.83-0.89$ \\
\hline Frailty prevalence & $2.30 \%$ & & $3.80 \%$ & \\
\hline Positive predictive value & $12.49 \%$ & $9.24-16.66 \%$ & $21.74 \%$ & $17.71-26.40 \%$ \\
\hline Negative predictive value & $97.87 \%$ & $97.82-97.93 \%$ & $96.72 \%$ & $96.61-96.84 \%$ \\
\hline \multirow[t]{2}{*}{ Accuracy } & $96.44 \%$ & $95.92-96.90 \%$ & $94.60 \%$ & $93.98-95.17 \%$ \\
\hline & \multicolumn{2}{|c|}{ Clinical frailty scale } & \multicolumn{2}{|c|}{ Frailty index } \\
\hline Sensitivity & $26.57 \%$ & $23.04-30.32 \%$ & $33.33 \%$ & $29.54-37.29 \%$ \\
\hline Specificity & $90.92 \%$ & $90.10-91.69 \%$ & $89.59 \%$ & $88.72-90.41 \%$ \\
\hline Positive likelihood ratio & 2.93 & $2.49-3.43$ & 3.2 & $2.78-3.68$ \\
\hline Negative likelihood ratio & 0.81 & $0.77-0.85$ & 0.74 & $0.70-0.79$ \\
\hline Frailty prevalence & $10.90 \%$ & & $12.80 \%$ & \\
\hline Positive predictive value & $26.35 \%$ & $23.37-29.57 \%$ & $31.97 \%$ & $29.01-35.08 \%$ \\
\hline Negative predictive value & $91.01 \%$ & $90.60-91.40 \%$ & $90.15 \%$ & $89.63-90.65 \%$ \\
\hline Accuracy & $83.90 \%$ & $82.92-84.85 \%$ & $82.39 \%$ & $81.37-83.37 \%$ \\
\hline
\end{tabular}

not classified as frail by any tool was $7.0 \%$ (Table 1). Table 2 shows the proportions for those aged $\geq 65$.

The calculated sensitivities, specificities and associated diagnostic statistics for 8-year mortality according to each frailty tool are shown in Table 3 (aged $\geq 50$ ). In general, frailty classifications had high specificity, that is, the probabilities of the tests predicting survival when baseline frailty was not identified were high (from highest to lowest: FRAIL 98.5\%, FP 97.7\%, CFS 90.9\% and FI 89.6\%). However, sensitivities (probability of the test predicting death when frailty was identified) were generally low (from highest to lowest: FI, 33.3\%, CFS 26.6\%, FP 16.2\% and FRAIL 9.1\%). Adjusted by the corresponding frailty prevalences, negative predictive values were high (and ordered the same as specificities from highest to lowest: FRAIL 97.9\%, FP 96.7\%, CFS $91.0 \%$ and FI 90.2\%), and positive predictive values were low (and ordered the same as sensitivities from highest to lowest: FI, 32.0\%, CFS 26.4\%, FP 21.7\% and FRAIL
$12.5 \%$ ). In terms of accuracy (overall probability that a participant was correctly classified), values were, from highest to lowest: FRAIL 96.4\%, FP 94.6\%, CFS 83.9\%, and FI $82.4 \%$. Table 4 shows the results for those aged $\geq 65$.

Table 1 shows the result of the binary logistic regression models in those aged $\geq 50$. The highest adjusted OR for mortality was for FRAIL (OR 4.48, 95\% CI 2.93-6.85, $P<0.001$ ), followed by FP (OR 3.55, 95\% CI 2.52-5.00, $P<0.001$ ), FI (OR 2.10, 95\% CI 1.68-2.62, $P<0.001$ ), and CFS (OR 1.88, 95\% CI 1.48-2.38, $P<0.001$ ). Table 2 shows the results for those aged $\geq 65$.

\section{Discussion}

In the present study, we compared the ability of four different frailty identification tools to predict 8-year mortality in TILDA. All four frailty tools significantly predicted 
Table 4 Diagnostic evaluation of the four frail classifications for the prediction of 8-year mortality in TILDA (population aged 65 and over, $N=2289$ )

\begin{tabular}{|c|c|c|c|c|}
\hline & Value & $95 \% \mathrm{CI}$ & Value & $95 \% \mathrm{CI}$ \\
\hline & \multicolumn{2}{|l|}{ FRAIL } & \multicolumn{2}{|c|}{ Frailty phenotype } \\
\hline Sensitivity & $10.08 \%$ & $7.53-13.15 \%$ & $18.70 \%$ & $15.29-22.50 \%$ \\
\hline Specificity & $98.01 \%$ & $97.26-98.61 \%$ & $96.41 \%$ & $95.45-97.22 \%$ \\
\hline Positive likelihood ratio & 5.08 & $3.34-7.73$ & 5.22 & $3.85-7.06$ \\
\hline Negative likelihood ratio & 0.92 & $0.89-0.95$ & 0.84 & $0.81-0.88$ \\
\hline Frailty prevalence & $3.70 \%$ & & $6.70 \%$ & \\
\hline Positive predictive value & $16.33 \%$ & $11.36-22.90 \%$ & $27.25 \%$ & $21.66-33.66 \%$ \\
\hline Negative predictive value & $96.60 \%$ & $96.49-96.70 \%$ & $94.29 \%$ & $94.05-94.52 \%$ \\
\hline \multirow[t]{2}{*}{ Accuracy } & $94.76 \%$ & $93.77-95.64 \%$ & $91.21 \%$ & $89.97-92.34 \%$ \\
\hline & \multicolumn{2}{|c|}{ Clinical frailty scale } & \multicolumn{2}{|c|}{ Frailty index } \\
\hline Sensitivity & $29.41 \%$ & $25.35-33.73 \%$ & $37.61 \%$ & $33.24-42.13 \%$ \\
\hline Specificity & $86.76 \%$ & $85.12-88.29 \%$ & $82.13 \%$ & $80.29-83.87 \%$ \\
\hline Positive likelihood ratio & 2.22 & $1.85-2.67$ & 2.1 & $1.81-2.45$ \\
\hline Negative likelihood ratio & 0.81 & $0.77-0.86$ & 0.76 & $0.71-0.82$ \\
\hline Frailty prevalence & $16.60 \%$ & & $22.00 \%$ & \\
\hline Positive predictive value & $30.66 \%$ & $26.93-34.67 \%$ & $37.25 \%$ & $33.76-40.86 \%$ \\
\hline Negative predictive value & $86.06 \%$ & $85.32-86.78 \%$ & $82.35 \%$ & $81.27-83.39 \%$ \\
\hline Accuracy & $77.24 \%$ & $75.47-78.95 \%$ & $72.33 \%$ & $70.45-74.16 \%$ \\
\hline
\end{tabular}

Calculations performed in Software Ltd. Diagnostic test evaluation calculator. https://www.medcalc.org/calc/diagnostic_test.php (Version 20.009; accessed September 11, 2021)

8-year mortality, but FRAIL and FP seemed more specific and FI and CFS a little more sensitive, although sensitivities were generally very low. Consequently, in the context of a population-based study where the general mortality proportion and prevalences of frailty are low, the use of a frailty identification tool for the specific purpose of the prediction of 8-year mortality could be useful to rule out the outcome when frailty is not identified at baseline, rather than to predict the outcome when frailty is identified. In this regard, the most accurate tool to rule out 8-year mortality was the negative classification by FRAIL, followed by the negative classification by FP (Tables 3 and 4).

Our results are in keeping with those of other population-based studies. In HRS, researchers cross-sectionally compared three models of frailty (functional domains, burden model and biologic syndrome), and also noted that a very small proportion of participants $(3.1 \%)$ were frail according to all three models, with a significant overlap in the proportions of participants classified as frail [3]. In NHANES, researchers also found that the prevalence of frailty was lower using the FP approach (3.6\%) compared to the FI (34\%) [4]. In SHARE, researchers found areas under the curve (AUC) for the prediction of 5-year mortality of 0.67 (95\% CI $0.65-0.68)$ for the FRAIL scale, 0.70 (0.68-0.71) for the FP, $0.70(0.68-0.71)$ for the CFS, and $0.75(0.74-0.77)$ for the FI, which is consistent with our result that FRAIL had the lowest and FI the highest PPV, although in their study AUCs for FP and CFS were indistinguishable [5]. In the Tree-City study, the 10-year mortality AUC was also lower for FP (0.56 [0.52-0.60]) than for FI (0.63 [0.60-0.67]) [6]. In a large comparative study, ELSA researchers noted that fully adjusted 3.5-year mortality hazard ratios (HRs) for frailty states varied depending on the classification used: 1.5 (95\% CI 0.8-3.0) for FRAIL, 1.8 (0.7-4.4) for FP, and 2.3 (1.5-3.5) for a CGA-based FI [7], also in keeping with our PPV results.

Given that the gold standard for the assessment and management of frailty in an individual is the provision of comprehensive geriatric assessment (CGA), which is time and resource intensive, it would appear reasonable (from the simplistic perspective of aiming to lower the 8-year mortality risk in the population) that a negative result in either FRAIL or FP would be used to rule out CGA referral, and a positive result in any tool used to rule in CGA referral, with a prioritisation as follows according to positive predictive values (PPV), from most to least urgent: FI, CFS, FP and FRAIL (this order of decreasing PPV values applies to both $\geq 50$ and $\geq 65$ populations as per Tables 3 and 4). Naturally, this recommendation is more theoretical than practical, because CGA indication is not only the reduction of mortality risk, but also the preservation of functional decline and promotion of independent living [15]. In addition, this theoretical scheme could be difficult to implement in real practice since we found overlaps in the proportions 
of participants classified as frail by the four tools (Figs. 1 and 2). However, the results of our study help appreciate that different frailty tools have different diagnostic properties and could be used differently in population screening programmes and clinical pathways.

Author contributions All authors listed have made a substantial, direct, and intellectual contribution to the work and approved it for publication.

Funding Roman Romero-Ortuno is funded by a Grant from Science Foundation Ireland under Grant number 18/FRL/6188. TILDA is funded by Atlantic Philanthropies, Irish Life, and the Irish Department of Health. Peter Hartley is funded by a fellowship from the Cambridge Biomedical Research Centre and The Addenbrooke's Charitable Trust [grant reference: $03 / 20 \mathrm{~A}$ ]. The funders had no role in the conduct of the research and/or preparation of the article; in study design; in the collection, analysis, and interpretation of data; in writing of the report; or in the decision to submit the paper for publication.

\section{Declarations}

\section{Conflict of interest None.}

Ethical approval Ethical approval for each wave was obtained from the Faculty of Health Sciences Research Ethics Committee at Trinity College Dublin, Ireland.

Informed consent All participants provided written informed consent.

Open Access This article is licensed under a Creative Commons Attribution 4.0 International License, which permits use, sharing, adaptation, distribution and reproduction in any medium or format, as long as you give appropriate credit to the original author(s) and the source, provide a link to the Creative Commons licence, and indicate if changes were made. The images or other third party material in this article are included in the article's Creative Commons licence, unless indicated otherwise in a credit line to the material. If material is not included in the article's Creative Commons licence and your intended use is not permitted by statutory regulation or exceeds the permitted use, you will need to obtain permission directly from the copyright holder. To view a copy of this licence, visit http://creativecommons.org/licenses/by/4.0/.

\section{References}

1. Clegg A, Young J, Iliffe S, Rikkert MO, Rockwood K (2013) Frailty in elderly people. Lancet 381(9868):752-762

2. Faller JW, Pereira DDN, de Souza S, Nampo FK, Orlandi FS, Matumoto S (2019) Instruments for the detection of frailty syndrome in older adults: a systematic review. PLoS ONE 14(4): 0216166

3. Cigolle CT, Ofstedal MB, Tian Z, Blaum CS (2009) Comparing models of frailty: the Health and Retirement Study. J Am Geriatr Soc 57(5):830-839

4. Blodgett J, Theou O, Kirkland S, Andreou P, Rockwood K (2015) Frailty in NHANES: comparing the frailty index and phenotype. Arch Gerontol Geriatr 60(3):464-470

5. Theou O, Brothers TD, Mitnitski A, Rockwood K (2013) Operationalization of frailty using eight commonly used scales and comparison of their ability to predict all-cause mortality. J Am Geriatr Soc 61(9):1537-1551

6. Gonzalez-ColacoHarmand M, Meillon C, Bergua V, TabueTeguo M, Dartigues JF, Avila-Funes JA et al (2017) Comparing the predictive value of three definitions of frailty: results from the ThreeCity study. Arch Gerontol Geriatr 72:153-163

7. Aguayo GA, Vaillant MT, Donneau AF, Schritz A, Stranges S, Malisoux L et al (2018) Comparative analysis of the association between 35 frailty scores and cardiovascular events, cancer, and total mortality in an elderly general population in England: an observational study. PLoS Med 15(3):1002543

8. Donoghue OA, McGarrigle CA, Foley M, Fagan A, Meaney J, Kenny RA (2018) Cohort profile update: The Irish Longitudinal Study on Ageing (TILDA). Int J Epidemiol 47(5):1398-13981

9. Fried LP, Tangen CM, Walston J, Newman AB, Hirsch C, Gottdiener J et al (2001) Frailty in older adults: evidence for a phenotype. J Gerontol A Biol Sci Med Sci 56(3):M146-M156

10. Romero-Ortuno R, Scarlett S, O'Halloran AM, Kenny RA (2019) Is phenotypical prefrailty all the same? A longitudinal investigation of two prefrailty subtypes in TILDA. Age Ageing 49(1):39-45

11. O’Halloran AM, Laird EJ, Feeney J, Healy M, Moran R, Beatty S et al (2020) Circulating micronutrient biomarkers are associated with 3 measures of frailty: evidence from the irish longitudinal study on ageing. J Am Med Dir Assoc 21(2):240-247

12. Roe L, Normand C, Wren MA, Browne J, O'Halloran AM (2017) The impact of frailty on healthcare utilisation in Ireland: evidence from the Irish longitudinal study on ageing. BMC Geriatr 17(1):203

13. O'Halloran AM, Hartley P, Moloney D, McGarrigle C, Kenny RA, Romero-Ortuno R (2021) Informing patterns of health and social care utilisation in Irish older people according to the Clinical Frailty Scale. HRB Open Res 4:54

14. Ward M, May P, Briggs R, McNicholas T, Normand C, Kenny RA et al (2020) Linking death registration and survey data: procedures and cohort profile for The Irish Longitudinal Study on Ageing (TILDA). HRB Open Res 3:43

15. Garrard JW, Cox NJ, Dodds RM, Roberts HC, Sayer AA (2020) Comprehensive geriatric assessment in primary care: a systematic review. Aging Clin Exp Res 32(2):197-205

Publisher's Note Springer Nature remains neutral with regard to jurisdictional claims in published maps and institutional affiliations. 